\title{
Cytotoxic Constituents from the Roots of Asarum sieboldii in Human Breast Cancer Cells
}

\author{
Eunae Kim ${ }^{1}$, Hyun Jung Kim² ${ }^{2}$ Ha-Na Oh${ }^{2}$, Ah-Won Kwak ${ }^{2}$, Su-Nam Kim ${ }^{3}$, Bok Yun Kang ${ }^{4}$, Seung-Sik Cho ${ }^{2}$, \\ Jung-Hyun Shim ${ }^{2, *}$, and Goo Yoon ${ }^{2, *}$ \\ ${ }^{1}$ College of Pharmacy, Chosun University, Gwangju 61452, Republic of Korea \\ ${ }^{2}$ College of Pharmacy, Mokpo National University, Muan, Jeonnam 58554, South Korea \\ ${ }^{3}$ KIST Gangneung Institute, Gangneung 25451, Republic of Korea \\ ${ }^{4}$ College of Pharmacy, Chonnam National University, Gwangju 61186, Republic of Korea
}

\begin{abstract}
Bioassay-guided fractionation of the roots of Asarum sieboldii led to the isolation of the six compounds methylkakuol (1), sesamin (2), asarinin (3), xanthoxylol (4), and (2E,4E,8Z,10E/Z)- $N$-(2-methylpropyl) dodeca-2,4,8,10-tetraenamide (5/6). Among the isolates, xanthoxylol (4) exhibited significant cytotoxicity against human breast cancer cells MCF-7 and MDA-MB-231 in vitro with $\mathrm{IC}_{50}$ values of 9.15 and $13.95 \mu \mathrm{M}$, respectively. Keywords - Asarum sieboldii, Cytotoxicity, Breast cancer, Xanthoxylol
\end{abstract}

\section{Introduction}

Breast cancer declared to occupy the highest incidence rate and to females among all cancers. Breast cancer leads to death more often than other cancers. In principle, breast cancer occurs from the imbalance of hormones by many risk factors. ${ }^{1}$ Breast cancer is classified into various molecular subtypes including estrogen receptor (ER), progesterone receptor (PR), and human epidermal growth factor receptor 2 (HER2). Approximately 15 to $20 \%$ of patients with breast cancer are ER(-), PR(-), and HER2(-) known as the triple negative breast cancer (TNBC). ${ }^{2}$ For the therapy of aggressive TNBC with distant recurrence and high rates of metastases, surgery is principle therapy, and chemotherapy is also needed to remove the remaining cancer cells. ${ }^{3}$ Minimizing surgical therapy and overcoming side effects are important in cancer treatment, and natural compounds have provided considerable number of clinically used anticancer drugs or lead compounds with potential for cancer therapy and drug development.

The Asarum sieboldii (Aristolochiaceae) is herbal plant distributed through East Asia. The roots of A. sieboldii

\footnotetext{
*Author for correspondence

Jung-Hyun Shim, College of Pharmacy, Mokpo National University,

Muan, Jeonnam 58554, South Korea

Tel: +82-61-450-2684; E-mail: s1004jh@gmail.com
}

Goo Yoon, College of Pharmacy, Mokpo National University, Muan, Jeonnam 58554, South Korea

Tel: +82-61-450-2682; E-mail: gyoon@mokpo.ac.kr were used in traditional medicine as antitussive, antiallergic, antihyperlipemic, expectorant, anti-inflammatory, anesthetic, and antifungal agent. ${ }^{4}$ In our previous study, we found that the $\mathrm{EtOH}$ extract of $A$. sieboldii exhibited PTP1B inhibitory effect. ${ }^{5}$ PTP1B plays a critical role in regulating body weight, glucose homeostasis by acting as a key negative regulator of insulin and leptin signaling pathway and also has recently drawn attention as an attractive target for anticancer, especially for the treatment of breast cancer. ${ }^{6}$ PTP1B is overexpressed in human breast cancer, and inhibition of PTP1B delays erbB2induced mammary tumorigenesis and protects from lung metastasis. ${ }^{7,8}$ So we screened the cytotoxicity of EtOH extract of $A$. sieboldii in human breast cancer cell lines and found its moderate anticancer effect. Accordingly, we investigated the bioassay-guided isolation and cytotoxic properties of six known isolates (1 - 6) from A. sieboldii on breast cancer cells including the TNBC MDA-MB-231 and the $\mathrm{ER}(+)$ non-TNBC MCF-7 breast cancer cell lines.

\section{Experimental}

General experimental procedures - IR spectra were recorded on a Nicolet 520P spectrometer (Polaris/ICON). EI-MS spectra were obtained on a GC (HP6890N)-MS (Agilent 5973N) spectrometer. UV spectrum was determined with a JASCO V-530 spectrophotometer. Nuclear magnetic resonance (NMR) spectra for ${ }^{1} \mathrm{H}$, and ${ }^{13} \mathrm{C}$ were taken on 
Bruker $400 \mathrm{MHz}$ spectrometer. Chemical shifts were expressed in parts per million (ppm) relative to TMS as the internal standard and coupling constants $(J)$ were given in $\mathrm{Hz}$. TLC was carried out on Merck silica gel $\mathrm{F}_{254}$ precoated glass plates. Flash chromatography (FC) and preparative HPLC was carried out with Isolera ${ }^{\mathrm{TM}}$ Spektra system and Waters 600 HPLC system, respectively.

Plant materials-The roots of $A$. sieboldii were purchased from Chonnam Herb Association and identified by Prof. Hyun Jung Kim from Mokpo National University, Muan, Korea. A voucher specimen (MNUYG010) was deposited in the College of Pharmacy, Mokpo National University, Muan, Korea.

Extraction and isolation - The roots of $A$. sieboldii $(1.2 \mathrm{~kg})$ were extracted twice with EtOH $(1 \mathrm{~L})$ for $3 \mathrm{~h}$ by sonication. The solvent was evaporated in vacuo to afford an $\mathrm{EtOH}$ residue $(91 \mathrm{~g})$, which was suspended in $\mathrm{H}_{2} \mathrm{O}(1 \mathrm{~L})$, and extracted with ethyl acetate (EA). The evaporation residue of EA fraction $(5 \mathrm{~g})$ was subjected to flash silica gel chromatography (FC), using $n$-hexane/EA solvent system (1:0 0:1), to afford 5 fractions. Compound 1 (150 $\mathrm{mg}$ ) was isolated from Fraction 2, which was subjected to FC, using $n$-hexane:EA solvent system (10:1). Fraction 3 was subjected to FC eluted gradient solvent mixtures with hexane/EA (10:1 8:1) to provide compounds 2 (120 mg) and $3(200 \mathrm{mg})$. Fraction 4 was purified by FC using $n$ hexane:EA solvent system $(7: 1)$ to afford a mixture of compounds 5 and $6(500 \mathrm{mg})$. Isomeric compounds 5/6 were further separated by chiral supercritical fluid chromatography (SFC) using Chiralpak IG (Daicel Chiral Technologies). Fraction 4 was further fractionated by FC eluting with gradient solvent system of hexane/EA (8:1 5:1) to yield compound $\mathbf{4}$ (40 mg). Compounds $\mathbf{1}$ - $\mathbf{4}$ were further purified by preparative HPLC using SunFire C18 column $(5 \mu \mathrm{m}, 150 \times 19 \mathrm{~mm}$, Waters $)$ with a gradient of $5 \%$ to $40 \%$ acetonitrile.

Methylkakuol (1) - White amorphous powder; ${ }^{1} \mathrm{H}-$ NMR (400 MHz, $\left.\mathrm{CDCl}_{3}\right): \delta 7.25(1 \mathrm{H}, \mathrm{s}, \mathrm{H}-6), 6.48(1 \mathrm{H}, \mathrm{s}$, $\mathrm{H}-3), 5.92\left(2 \mathrm{H}, \mathrm{s}, \mathrm{OCH}_{2} \mathrm{O}\right) 3.81\left(3 \mathrm{H}, \mathrm{s}, \mathrm{OCH}_{3}\right), 2.90(2 \mathrm{H}$, q, $J=7.2 \mathrm{~Hz}, \mathrm{H}-8), 1.10(3 \mathrm{H}, \mathrm{t}, J=7.2 \mathrm{~Hz}, \mathrm{H}-9) \cdot{ }^{13} \mathrm{C}-$ NMR (100 MHz, $\left.\mathrm{CDCl}_{3}\right): \delta 201.4(\mathrm{C}-7), 157.6(\mathrm{C}-2)$, 153.0 (C-4), 142.4 (C-5), 121.3 (C-1), 109.9 (C-6), 102.8 $\left(\mathrm{OCH}_{2} \mathrm{O}\right), 95.0(\mathrm{C}-3), 57.1\left(\mathrm{OCH}_{3}\right), 37.8(\mathrm{C}-8), 9.5(\mathrm{C}-9)$.

Sesamin (2) - White amorphous powder; ${ }^{1} \mathrm{H}-\mathrm{NMR}$ $\left(400 \mathrm{MHz}, \mathrm{CDCl}_{3}\right): \delta 6.87-6.78(6 \mathrm{H}, \mathrm{m}, \mathrm{Ar}-\mathrm{H}), 5.96$ $\left(4 \mathrm{H}, \mathrm{s}, \mathrm{OCH}_{2} \mathrm{O} \times 2\right) 4.73\left(2 \mathrm{H}, \mathrm{d}, J=4.0 \mathrm{~Hz}, \mathrm{H}-7,7^{\prime}\right), 4.24$ ( $2 \mathrm{H}$, dd, $\left.J=8.8,7.2 \mathrm{~Hz}, \mathrm{H}-9,9^{\prime}\right), 3.88$ (2H, dd, $J=9.2$, $\left.3.6 \mathrm{~Hz}, \mathrm{H}-9,99^{\prime}\right), 3.06$ (2H, m, H-8, 8'). ${ }^{13} \mathrm{C}-\mathrm{NMR}(100$ $\left.\mathrm{MHz}, \mathrm{CDCl}_{3}\right): \delta 148.0\left(\mathrm{C}-3,3^{\prime}\right), 147.1\left(\mathrm{C}-4,4^{\prime}\right), 135.1$ (C-1, 1'), 119.4 (C-6, 6'), 108.2 (C-5, 5'), 106.5 (C-2, 2'),
$101.1\left(\mathrm{OCH}_{2} \mathrm{O}\right), 85.8\left(\mathrm{C}-7,7^{\prime}\right), 71.7\left(\mathrm{C}-9,9^{\prime}\right), 54.3\left(\mathrm{C}-8,8^{\prime}\right)$.

Asarinin (3) - White amorphous powder; ${ }^{1} \mathrm{H}-\mathrm{NMR}$ $\left(400 \mathrm{MHz}, \mathrm{CDCl}_{3}\right): \delta 6.88-6.78(6 \mathrm{H}, \mathrm{m}, \mathrm{Ar}-\mathrm{H}), 5.97$ $\left(2 \mathrm{H}, \mathrm{s}, \mathrm{OCH}_{2} \mathrm{O}\right), 5.96\left(2 \mathrm{H}, \mathrm{s}, \mathrm{OCH}_{2} \mathrm{O}\right), 4.84(1 \mathrm{H}, \mathrm{d}$, $J=5.2 \mathrm{~Hz}, \mathrm{H}-7), 4.41\left(1 \mathrm{H}, \mathrm{d}, J=7.2 \mathrm{~Hz}, \mathrm{H}-7^{\prime}\right), 4.11(1 \mathrm{H}$, d, $J=9.6 \mathrm{~Hz}, \mathrm{H}-9), 3.84$ (2H, m, H-9, 9'), $3.32(2 \mathrm{H}, \mathrm{m}$, $\left.\mathrm{H}-8,9^{\prime}\right), 2.87\left(1 \mathrm{H}, \mathrm{m}, \mathrm{H}-8^{\prime}\right) .{ }^{13} \mathrm{C}-\mathrm{NMR}\left(100 \mathrm{MHz}, \mathrm{CDCl}_{3}\right)$ : $\delta 148.9$ (C-3'), 148.5 (C-4'), 148.1 (C-3), 147.5 (C-4), 136.0 (C-1), 133.1 (C-1'), 120.5 (C-6), 119.6 (C-6'), 109.1 (C-5, 5'), $107.5\left(\mathrm{C}-2^{\prime}\right), 107.3(\mathrm{C}-2), 102.0\left(\mathrm{OCH}_{2} \mathrm{O}\right)$, $101.9\left(\mathrm{OCH}_{2} \mathrm{O}\right), 88.5(\mathrm{C}-7), 82.9\left(\mathrm{C}-7^{\prime}\right), 71.8\left(\mathrm{C}-9^{\prime}\right), 70.6$ (C-9), 55.5 (C-8), 51.0 (C-8').

Xanthoxylol (4) - White amorphous powder; ${ }^{1} \mathrm{H}-\mathrm{NMR}$ $\left(400 \mathrm{MHz}, \mathrm{CDCl}_{3}\right): \delta 6.93-6.81(6 \mathrm{H}, \mathrm{m}, \mathrm{Ar}-\mathrm{H}), 5.99(2 \mathrm{H}$, $\left.\mathrm{s}, \mathrm{OCH}_{2} \mathrm{O}\right), 4.86\left(1 \mathrm{H}, \mathrm{d}, J=5.2 \mathrm{~Hz}, \mathrm{H}-7^{\prime}\right), 4.41(1 \mathrm{H}, \mathrm{d}$, $J=7.2 \mathrm{~Hz}, \mathrm{H}-7), 4.13(1 \mathrm{H}, \mathrm{d}, J=9.6 \mathrm{~Hz}, \mathrm{H}-9), 3.94(3 \mathrm{H}$, s, $\left.\mathrm{OCH}_{3}\right), 3.84\left(2 \mathrm{H}, \mathrm{m}, \mathrm{H}-9,9^{\prime}\right), 3.34$ (2H, m, H-8, 9'), $2.93\left(1 \mathrm{H}, \mathrm{m}, \mathrm{H}-8^{\prime}\right) .{ }^{13} \mathrm{C}-\mathrm{NMR}\left(100 \mathrm{MHz}, \mathrm{CDCl}_{3}\right): \delta 147.7$ (C-3'), 146.8 (C-4'), 146.5 (C-3), 145.4 (C-4), 132.9 (C$\left.1^{\prime}\right), 132.2$ (C-1), 119.2 (C-6'), 118.7 (C-6), 114.3 (C-5), 108.6 (C-5'), 108.2 (C-2), $106.4\left(\mathrm{C}-2^{\prime}\right), 101.0\left(\mathrm{OCH}_{2} \mathrm{O}\right)$, 87.8 (C-7'), 82.1 (C-7), 70.9 (C-9'), 69.6 (C-9), 55.9 (C$\left.8^{\prime}\right), 54.5\left(\mathrm{OCH}_{3}\right), 50.1(\mathrm{C}-8)$.

$(2 E, 4 E, 8 Z, 10 E)-N$-(2-methylpropyl)dodeca-2,4,8,10tetraenamide (5) - White amorphous powder; ${ }^{1} \mathrm{H}-\mathrm{NMR}$ $\left(400 \mathrm{MHz}, \mathrm{CDCl}_{3}\right): \delta 7.12(1 \mathrm{H}, \mathrm{dd}, J=14.8,10.4 \mathrm{~Hz}, \mathrm{H}-$ 3), $6.30(1 \mathrm{H}$, br t, $J=12.0 \mathrm{~Hz}, \mathrm{H}-10), 6.09(1 \mathrm{H}, \mathrm{dd}$, $J=15.2,10.4 \mathrm{~Hz}, \mathrm{H}-4), 6.00$ (1H, dt, $J=15.2,6.4 \mathrm{~Hz}, \mathrm{H}-$ 5), $5.97(1 \mathrm{H}, \mathrm{t}, J=10.8 \mathrm{~Hz}, \mathrm{H}-9), 5.69(1 \mathrm{H}, \mathrm{d}, J=14.8$ $\mathrm{Hz}, \mathrm{H}-2), 5.63(1 \mathrm{H}, \mathrm{dd}, J=14.8,7.2 \mathrm{~Hz}, \mathrm{H}-11), 5.58(1 \mathrm{H}$, br s, NH), $5.20(1 \mathrm{H}, \mathrm{dt}, J=10.8,6.8 \mathrm{~Hz}, \mathrm{H}-8), 3.09(2 \mathrm{H}$, t, $\left.J=6.8 \mathrm{~Hz}, \mathrm{H}-1^{\prime}\right), 2.23-2.14(4 \mathrm{H}, \mathrm{m}, \mathrm{H}-6,7), 1.80(1 \mathrm{H}$, m, H-2') $1.71(3 \mathrm{H}, \mathrm{d}, J=6.8 \mathrm{~Hz}, \mathrm{H}-12), 0.85(6 \mathrm{H}, \mathrm{d}$, $\left.J=6.8 \mathrm{~Hz}, \mathrm{H}-3^{\prime}, 4^{\prime}\right) .{ }^{13} \mathrm{C}-\mathrm{NMR}\left(100 \mathrm{MHz}, \mathrm{CDCl}_{3}\right): \delta$ 165.3 (C-1), 141.0 (C-5), 140.1 (C-3), 128.8 (C-11), 128.3 (C-9), 127.6 (C-4), 126.9 (C-8), 125.7 (C-10), 121.1 (C2), 45.9 (C-1'), 32.0 (C-6), 28.7 (C-2'), 25.9 (C-7), 19.1 (C-3', 4'), 17.3 (C-12).

$(2 E, 4 E, 8 Z, 10 Z)-N$-(2-methylpropyl)dodeca-2,4,8,10tetraenamide (6) - White amorphous powder; ${ }^{1} \mathrm{H}-\mathrm{NMR}$ $\left(400 \mathrm{MHz}, \mathrm{CDCl}_{3}\right): \delta 7.12(1 \mathrm{H}, \mathrm{dd}, J=14.8,10.4 \mathrm{~Hz}, \mathrm{H}-$ 3), $6.24(1 \mathrm{H}$, br t, $J=10.4 \mathrm{~Hz}, \mathrm{H}-10), 6.21-6.12(1 \mathrm{H}, \mathrm{m}$, $\mathrm{H}-4), 6.08-5.96(2 \mathrm{H}, \mathrm{m}, \mathrm{H}-5,9), 5.71(1 \mathrm{H}, \mathrm{d}, J=14.8 \mathrm{~Hz}$, $\mathrm{H}-2), 5.54(1 \mathrm{H}$, br s, NH), $5.50(1 \mathrm{H}, \mathrm{dd}, J=7.6,2.4 \mathrm{~Hz}$, $\mathrm{H}-11), 5.20(1 \mathrm{H}, \mathrm{dt}, J=9.6,6.4 \mathrm{~Hz}, \mathrm{H}-8), 3.08(2 \mathrm{H}, \mathrm{br}$ t, $\left.J=4.4 \mathrm{~Hz}, \mathrm{H}-1^{\prime}\right), 2.25-2.17$ (4H, m, H-6, 7), $1.75(1 \mathrm{H}, \mathrm{m}$, H-2'), 1.68 (3H, d, $J=6.8 \mathrm{~Hz}, \mathrm{H}-12), 0.86(6 \mathrm{H}, \mathrm{d}, J=6.8$ $\left.\mathrm{Hz}, \mathrm{H}-3^{\prime}, 4^{\prime}\right) .{ }^{13} \mathrm{C}-\mathrm{NMR}\left(100 \mathrm{MHz}, \mathrm{CDCl}_{3}\right): \delta 166.7$ (C1), 142.4 (C-5), 141.5 (C-3), 130.5 (C-11), 129.1 (C-9), 127.2 (C-4), 124.6 (C-8), 124.5 (C-10), 122.5 (C-2), 47.3 
(C-1'), 33.3 (C-6), 30.1 (C-2'), 29.0 (C-7), 20.6 (C-3', 4'), 13.6 (C-12).

Cytotoxicity assay - Cell viability was measured with the dimethyl sulfoxide and 3-(4,5-dimethylthiazol-2-yl)2,5-diphenyltetrazolium bromide [MTT, Sigma-Aldrich (St. Louis, MO, USA)] assay. MCF-7 $\left(9 \times 10^{3}\right.$ cells/well $)$ and MDA-MB-231 $\left(3.5 \times 10^{3}\right.$ cells/well $)$ cells were seeded on a 96 -well microtiter plate after then they were treated with wide range of doses of compounds $\mathbf{1}$ - $\mathbf{6}$ for $48 \mathrm{~h}$. MTT solution $(5 \mathrm{mg} / \mathrm{mL})$ was added to the cells prior to incubation at $37^{\circ} \mathrm{C}$ for $1 \mathrm{~h}$. After supernatant removed, the formazan crystals were dissolved in $100 \mu \mathrm{L}$ dimethyl sulfoxide for $5 \mathrm{~min}$ at RT. The absorbance was measured at $570 \mathrm{~nm}$ using a spectrophotometer (Thermo Fisher Scientific, Vantaa, Finland). Percentages of compounds $\mathbf{1}$ - $\mathbf{6}$ treated cell viabilities were normalized to that of untreated cells.

Statistical analysis - Results were presented as mean \pm SD of at least three independent experiments performed in triplicates. Data were analyzed for statistical significance using a one-way analysis of variance. A $p$-value of less than 0.05 was considered statistically significant.

\section{Results and Discussion}

The air-dried $A$. sieboldii roots were extracted twice with $\mathrm{EtOH}$ for $3 \mathrm{~h}$ by sonication. The solvent was evaporated in vacuo to afford an $\mathrm{EtOH}$ residue, which was suspended in $\mathrm{H}_{2} \mathrm{O}$, and partitioned with ethyl acetate (EA). EA fraction was subjected to flash silica gel chromatography and preparative HPLC to obtain six known compounds 1 - $\mathbf{6}$ (Fig. 1). By comparison of their spectroscopic data with previously reported values in the literature, these compounds were confirmed as the essential oil, methylkakuol $(\mathbf{1})^{9}$, the lignan, sesamin $(\mathbf{2})^{10}$, asarinin
$(3)^{10}$, xanthoxylol $(4)^{11,12}$, and the alkylamide, $(2 E, 4 E, 8 Z$, $10 E)-N$-(2-methylpropyl)dodeca-2,4,8,10-tetraenamide

(5) and $(2 E, 4 E, 8 Z, 10 Z)-N$-(2-methylpropyl)dodeca-2,4,8, 10-tetraenamide $(\mathbf{6}){ }^{13,14}$

MTT assay was performed to determine the effect of six known isolates $(\mathbf{1}-\mathbf{6})$ on the viability of human breast cancer cells MCF-7 and MDA-MB-231. The concentration of compounds 1-6 were decided after confirming its significant viability inhibition on breast cancer cells. In order to obtain $\mathrm{IC}_{50}$ values, dose-dependent treatment of 1 - 6 were performed in both MCF-7 and MDA-MB-231 cells. The essential oil, methylkakuol (1) and the alkylamide, $(2 E, 4 E, 8 Z, 10 E)$ - and $(2 E, 4 E, 8 Z, 10 Z)-N-(2-$ methylpropyl)dodeca-2,4,8,10-tetraenamides (5 and 6) were not cytotoxic on both MCF-7 and MDA-MB-231 cells. The lignans, sesamin (2) and asarinin (3) showed weak cytotoxicity with $\mathrm{IC}_{50}$ values of 98.57 and $67.25 \mu \mathrm{M}$, respectively, in MCF-7 cells, not in MDA-MB-231 cells. Among the isolates, xanthoxylol (4) exhibited significant cytotoxicity in both cell lines. Our results showed the decreased viability of MCF-7 and MDA-MB-231 cells in a dose-dependent manner. The $\mathrm{IC}_{50}$ values of inhibition of cell viability in MCF-7 cells was $9.15 \mu \mathrm{M}$ and MDAMB-231 cells was $13.95 \mu \mathrm{M}$ for $48 \mathrm{~h}$ xanthoxylol treatment. It has superior efficacy compared to 5-fluoruracil (5-FU) which is widely used as anticancer drug, and it shows possibility of new breast cancer treatment in the future (Table 1). Sesamin (2) and asarinin (3) are C-7' epimeric furofuran lignans with two benzo[1,3]dioxole. It is presumed that the difference of $\mathrm{IC}_{50}$ values between sesamin and asarininis caused by stereochemistry of C-7'. The structural difference of asarinin and xanthoxylol is the substituent of C-7. Benzo[1,3]dioxole in asarinin is replaced with 2-hydroxy-3-methoxybenzene in xanthoxylol. Therefore, we can assume that the hydroxyl group in

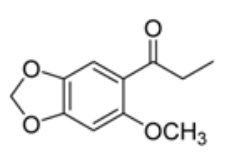

Methylkakuol (1)

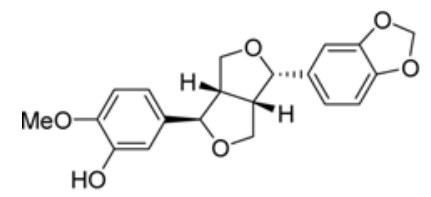

Xanthoxylol (4)
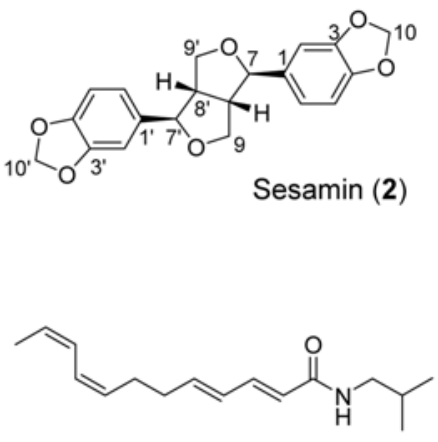

(2E,4E,8Z,10E)-N-(2-methylpropyl) dodeca-2,4,8,10-tetraenamide (5)
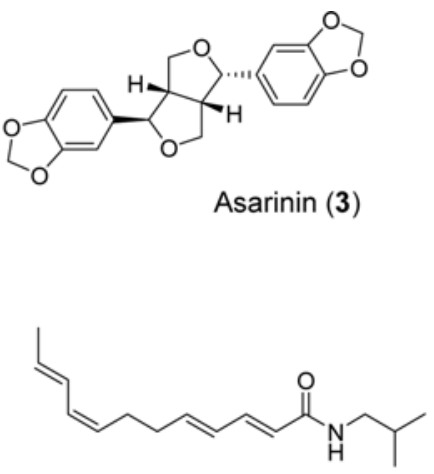

(2E,4E,8Z,10Z)-N-(2-methylpropyl) dodeca-2,4,8,10-tetraenamide (6)

Fig. 1. Structures of isolated compounds (1 - 6) from A. sieboldii. 
Table 1. The anti-proliferative effect of compounds (1 - 6) on human breast cancer cells (MCF-7 and MDA-MB-231).

\begin{tabular}{lcc}
\hline \hline \multicolumn{1}{c}{ Compounds } & MCF-7 $\left(\mathrm{IC}_{50}, \mu \mathrm{M}\right)$ & $\left.\mathrm{MDA}^{-\mathrm{MB}-231(\mathrm{IC}} \mathrm{C}_{50}, \mu \mathrm{M}\right)$ \\
\hline Methylkakoul (1) & $>100$ & $>100$ \\
Sesamin (2) & 98.57 & $>100$ \\
Asarinin (3) & 67.25 & $>100$ \\
Xanthoxylol (4) & 9.15 & 13.95 \\
$(2 E, 4 E, 8 Z, 10 E)-N$-(2-methylpropyl)dodeca-2,4,8,10-tetraenamide (5) & $>100$ & $>100$ \\
$(2 E, 4 E, 8 Z, 10 Z)-N$-(2-methylpropyl)dodeca-2,4,8,10-tetraenamide (6) & $>100$ & $>100$ \\
5-Fluorouracil & 42.09 & 27.19 \\
\hline
\end{tabular}

xanthoxylol plays an important role in terms of the cytotoxic efficacy on both human breast cancer cell lines, MCF-7 and MDA-MB-231.

In conclusion, we investigated the anticancer activity of compounds 1 - 6 from $A$. sieboldii against human breast cancer cell lines, MCF-7 and MDA-MB-231. Two lignans, sesamin (2) andasarin in (3) showed moderate cytotoxicity with $\mathrm{IC}_{50}$ values of 98.57 and $67.25 \mu \mathrm{M}$, respectively, only in MCF-7 cells. The lignin, xanthoxylol (4) exhibited significant cytotoxicity in both cell lines with $\mathrm{IC}_{50}$ values of 9.15 and $13.95 \mu \mathrm{M}$, respectively. Thus, the mechanisms of xanthoxylol and structure-activity relationship study of lignans are worthy of further investigation.

\section{Acknowledgments}

This research was supported by Research Funds of Mokpo National University in 2016.

\section{References}

(1) Siegel, R. L.; Miller, K. D.; Jemal, A. CA Cancer J. Clin. 2016, 66, 7-30.

(2) Donepudi, M. S.; Kondapalli, K.; Amos, S. J.; Venkanteshan, P. J. Cancer Res. Ther. 2014, 10, 506-511.

(3) Andreopoulou, E.; Schweber, S. J.; Sparano, J. A.; McDaid, H. M.
Expert Opin. Pharmacother. 2015, 16, 983-998.

(4) Han, A. R.; Kim, H. J.; Shin, M.; Hong, M.; Kim, Y. S.; Bae, H. Chem. Biodivers. 2008, 5, 346-351.

(5) Lee, W.; Kim, H. J.; Moon, H. S.; Kim, S. N.; Yoon, G. Kor. J. Pharmacogn. 2013, 44, 176-181.

(6) Tonks, N. K.; Muthuswamy, S. K. Cancer Cell 2007, 11, 214-216.

(7) Bjorge, J. D.; Pang, A.; Fujita, D. J. J. Biol. Chem. 2000, 275, 41439-41446.

(8) Julien, S. G.; Dubé, N.; Read, M.; Penney, J.; Paquet, M.; Han, Y.; Kennedy, B. P.; Muller, W. J.; Tremblay, M. L. Nat. Genet. 2007, 39, 338346.

(9) Reigada, J. B.; Tcacenco, C. M.; Andrade, L. H.; Kato, M. J.; Porto, A. L. M.; Lago, J. H. G. Tetrahedron-Asymmetr. 2007, 18, 1054-1058.

(10) Bussey, R. O. 3rd.; Sy-Cordero, A. A.; Figueroa, M.; Carter, F. S.; Falkinham, J. O. 3rd.; Oberlies, N. H.; Cech, N. B. Planta Med. 2014, 80, 498-501.

(11) Choi, H. G.; Choi, Y. H.; Kim, J. H.; Kim, H. H.; Kim, S. H.; Kim, J. A.; Lee, S. M.; Na, M.; Lee, S. H. Arch. Pharm. Res. 2014, 37, 467472.

(12) Swain, N. A.; Brown, R. C.; Bruton, G. A. J. Org. Chem. 2004, 69, 122-129.

(13) Yasuda, I.; Takeya, K.; Itokawa, H. Chem. Pharm. Bull. 1981, 29, 564-566.

(14) Matovic, N. J.; Hayes, P. Y.; Penman, K.; Lehmann, R. P.; De Voss, J. J. J. Org. Chem. 2011, 76, 4467-4481.
Received July 26, 2018

Revised November 29, 2018 Accepted December 1, 2018 